Jin-Long Yan*

\title{
Crystal structure of $\mathbf{N}$-(amino(pyrazin-2-yl)methylene)-6-methylpyridin-1- ium-3-carbohydrazonate- $\mathrm{K}^{3} \mathrm{O}, \mathrm{N}, \mathrm{N}^{\prime}$ )-(dinitrato-k $\mathrm{K}^{1} \mathrm{O}$ )zinc(II), $\mathrm{C}_{12} \mathrm{H}_{12} \mathrm{~N}_{8} \mathrm{O}_{7} \mathrm{Zn}$
}

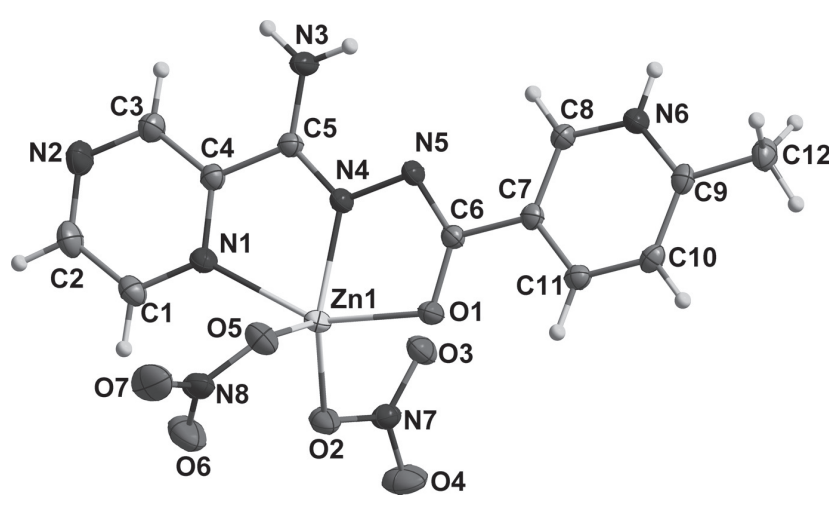

https://doi.org/10.1515/ncrs-2019-0605

Received August 21, 2019; accepted October 16, 2019; available online November 8, 2019

\section{Abstract}

$\mathrm{C}_{12} \mathrm{H}_{12} \mathrm{~N}_{8} \mathrm{O}_{7} \mathrm{Zn}$, monoclinic, $P 2_{1} / c$ (no. 14), $a=8.2116(3) \AA$, $b=11.1926(4) \AA, \quad c=17.3488(7) \AA, \quad \beta=96.2450(10)^{\circ}$, $V=1585.05(10) \AA^{3}, Z=4, R_{\mathrm{gt}}(F)=0.0263, w R_{\mathrm{ref}}\left(F^{2}\right)=0.0722$, $T=150(2) \mathrm{K}$.

CCDC no.: 1959581

The molecular structure of the title complex is shown in the figure. Table 1 contains crystallographic data and Table 2 contains the list of the atoms including atomic coordinates and displacement parameters.

\section{Source of material}

The hydrazone ligand, used in this study was prepared according to the literature method while using 6-methylnicotinic acid hydrazide instead of nicotinic acid hydrazide [3]. The crystals of the title complex suitable for the X-ray analysis were obtained by slow evaporation of the ethanol solution of the hydrazone ligand with zinc nitrate.

\section{Experimental details}

The structure was solved by direct methods and refined with the SHELX software package [1]. The hydrogen atoms were placed at calculated positions and refined as riding atoms.

\footnotetext{
*Corresponding author: Jin-Long Yan, School of Science and Technology, Jiaozuo Teachers college, Jiaozuo 454001, P.R. China, e-mail: jinlong_yan@163.com. https://orcid.org/0000-0003-23961499
}

Table 1: Data collection and handling.

\begin{tabular}{|c|c|}
\hline Crystal: & Yellow rod \\
\hline Size: & $0.24 \times 0.06 \times 0.04 \mathrm{~mm}$ \\
\hline Wavelength: & $\mathrm{Cu} \mathrm{K} \alpha$ radiation $(1.54178 \AA$ ) \\
\hline$\mu:$ & $2.75 \mathrm{~mm}^{-1}$ \\
\hline Diffractometer, scan mode: & Bruker APEX-II, $\varphi$ and $\omega$ \\
\hline$\theta_{\max }$, completeness: & $79.7^{\circ},>99 \%$ \\
\hline $\mathrm{N}(\mathrm{hkl})_{\text {measured }}, \mathrm{N}(\mathrm{hkl})_{\text {unique }}, R_{\text {int }}$ : & $23387,3416,0.030$ \\
\hline Criterion for $\mathrm{I}_{\mathrm{obs}}, \mathrm{N}(\mathrm{hkl})_{\mathrm{gt}}$ : & $\mathrm{I}_{\mathrm{obs}}>2 \sigma\left(\mathrm{I}_{\mathrm{obs}}\right), 3271$ \\
\hline$N(\text { param })_{\text {refined }}:$ & 254 \\
\hline Programs: & SHELX [1], Bruker [2] \\
\hline
\end{tabular}

Table 2: Fractional atomic coordinates and isotropic or equivalent isotropic displacement parameters $\left(\AA^{2}\right)$.

\begin{tabular}{lrrrr}
\hline Atom & $\boldsymbol{x}$ & $\boldsymbol{y}$ & $\boldsymbol{Z}$ & $\boldsymbol{U}_{\text {iso }}{ }^{*} \boldsymbol{U}_{\text {eq }}$ \\
\hline Zn1 & $0.44852(2)$ & $0.17713(2)$ & $0.32758(2)$ & $0.01909(8)$ \\
O1 & $0.32777(15)$ & $0.32880(10)$ & $0.36491(6)$ & $0.0219(2)$ \\
O2 & $0.29118(15)$ & $0.09741(12)$ & $0.24861(7)$ & $0.0269(3)$ \\
O3 & $0.14928(16)$ & $0.07285(11)$ & $0.34672(7)$ & $0.0285(3)$ \\
O4 & $0.03960(18)$ & $0.03693(16)$ & $0.22983(9)$ & $0.0439(4)$ \\
O5 & $0.61312(16)$ & $0.28718(12)$ & $0.28486(7)$ & $0.0298(3)$ \\
O6 & $0.6016(2)$ & $0.17558(16)$ & $0.18288(9)$ & $0.0461(4)$ \\
O7 & $0.79866(19)$ & $0.30241(16)$ & $0.20616(9)$ & $0.0453(4)$ \\
N1 & $0.63308(17)$ & $0.02162(12)$ & $0.35115(8)$ & $0.0204(3)$ \\
N2 & $0.8661(2)$ & $-0.14766(14)$ & $0.40783(10)$ & $0.0306(3)$ \\
N3 & $0.67811(18)$ & $0.11906(13)$ & $0.55206(8)$ & $0.0242(3)$ \\
H3A & 0.6408 & 0.1768 & 0.5798 & $0.029^{*}$ \\
H3B & 0.7521 & 0.0690 & 0.5737 & $0.029^{*}$ \\
N4 & $0.51369(17)$ & $0.17787(11)$ & $0.44165(8)$ & $0.0185(3)$ \\
N5 & $0.45611(16)$ & $0.26981(12)$ & $0.48502(7)$ & $0.0184(3)$ \\
N6 & $0.23729(17)$ & $0.54546(13)$ & $0.59109(8)$ & $0.0217(3)$ \\
H6 & 0.2518 & 0.5510 & 0.6420 & $0.026^{*}$ \\
N7 & $0.15462(17)$ & $0.06803(13)$ & $0.27606(8)$ & $0.0235(3)$ \\
N8 & $0.67324(18)$ & $0.25361(14)$ & $0.22309(8)$ & $0.0278(3)$ \\
C1 & $0.6964(2)$ & $-0.05515(15)$ & $0.30427(10)$ & $0.0247(3)$ \\
H1 & 0.6603 & -0.0537 & 0.2504 & $0.030^{*}$ \\
C2 & $0.8155(2)$ & $-0.13812(16)$ & $0.33288(11)$ & $0.0288(4)$ \\
H2 & 0.8616 & -0.1894 & 0.2974 & $0.035^{*}$ \\
C3 & $0.8006(2)$ & $-0.07065(15)$ & $0.45506(10)$ & $0.0256(3)$ \\
H3 & 0.8329 & -0.0750 & 0.5093 & $0.031^{*}$ \\
C4 & $0.68755(19)$ & $0.01493(14)$ & $0.42742(9)$ & $0.0185(3)$ \\
C5 & $0.62236(19)$ & $0.10750(13)$ & $0.47717(9)$ & $0.0177(3)$ \\
C6 & $0.36222(19)$ & $0.34009(13)$ & $0.43842(9)$ & $0.0172(3)$ \\
C7 & $0.28906(18)$ & $0.44268(13)$ & $0.47743(9)$ & $0.0170(3)$ \\
C8 & $0.30917(19)$ & $0.45412(14)$ & $0.55735(9)$ & $0.0201(3)$
\end{tabular}


Table 2 (continued)

\begin{tabular}{lrrrr}
\hline Atom & $\boldsymbol{x}$ & $\boldsymbol{y}$ & $\boldsymbol{z}$ & $\boldsymbol{U}_{\text {iso }} \boldsymbol{U}_{\text {eq }}$ \\
\hline H8 & 0.3734 & 0.3977 & 0.5883 & $0.024^{*}$ \\
C9 & $0.14376(19)$ & $0.62950(14)$ & $0.55108(9)$ & $0.0197(3)$ \\
C10 & $0.12549(19)$ & $0.62166(14)$ & $0.47121(9)$ & $0.0196(3)$ \\
H10 & 0.0633 & 0.6803 & 0.4413 & $0.024^{*}$ \\
C11 & $0.19699(19)$ & $0.52904(14)$ & $0.43443(9)$ & $0.0190(3)$ \\
H11 & 0.1833 & 0.5242 & 0.3794 & $0.023^{*}$ \\
C12 & $0.0640(2)$ & $0.72285(16)$ & $0.59524(11)$ & $0.0269(4)$ \\
H12A & 0.0093 & 0.6850 & 0.6364 & $0.040^{*}$ \\
H12B & -0.0170 & 0.7661 & 0.5601 & $0.040^{*}$ \\
H12C & 0.1472 & 0.7789 & 0.6183 & $0.040^{*}$ \\
\hline
\end{tabular}

\section{Comment}

Pyrazine hydrazones have been attracting much attention because of their strong tendency to chelate to transition metal ions, and their complexes exhibited amazing magnetic, photocatalysic and luminescent properties [3-5]. In this work, the zinc(II) complex of a substituted pyridine-pyrazinehydrazone ligand was synthesized and characterized by X-ray diffraction.

In the title crystal structure, the central zinc(II) ion is surrounded by a neutral hydrazone ligand (occuring in its ylid form; see the figure) with $\mathrm{ON}_{2}$ donor set, and two $\mathrm{O}$ atoms from two monodentate coordinating nitrato ligands. The $\mathrm{Zn}$ (II) possesses a distorted tetragonal pyramidal coordination geometry with the the geometric index $\tau$ value of 0.176 [6]. It should be noted that the $\mathrm{C}-\mathrm{O}$ distance of the hydrazone is 1.2822(19) $\AA$, indicating the enolation of the $\mathrm{C}=\mathrm{O}$ double bond [3]. Consequently, the terminal pydine $\mathrm{N}$ atom is protoned for charge balance. In the solid, intermolecular $\mathrm{N}-\mathrm{H} \cdots \mathrm{O}$ hydrogen bonds link the complexes into a two-dimensional supermolecular network parallel to the $b c$ plane.

\section{References}

1. Sheldrick, G. M.: Crystal refinement with SHELX. Acta Crystallogr. C71 (2015) 3-8.

2. Bruker. SMART and SAINT. Bruker AXS Inc., Madison, WI, USA (2007).

3. Xu, Z.-Q.; He, Y.-L.; Li, Q.-Q.; Zhang, P.-L.; Li, H.-J.; Wang, Y.: Three complexes based on pyridine-pyrazine-hydrazone ligand: syntheses, photocatalysis and luminescent properties. Chin. J. Inorg. Chem. 34 (2018) 757-766.

4. Shuvaev, K. V.; Dawe, L. N.; Thompson, L. K. A.: Mn" 12 dupramolecular array with four independent spin-coupled subunits. Eur. J. Inorg. Chem. 2010 (2010) 4583-4586.

5. Wu, G.; Luo, J.; Huang, W.; Huang, R.; Wu, D.: Solvolysiscontrolled chirality resolution throughmetal-chelation effect: from racemate to homochiral one-dimensional copper helicate. Inorg. Chem. Commun. 41 (2014) 25-28.

6. Ye, X.-P.; Wang, G.-J.; Pan, P.; Zhang, Z.-P.; Wu, W.-N.; Wang, Y.: Syntheses, crystal structures and biological activities of two $\mathrm{Cu}(\mathrm{II})$ complexes with an acylhydrazone ligand bearing pyrrole unit. Chin. J. Inorg. Chem. 30 (2014) 2789-2795. 